\title{
OSCILLATIONS IN REFLECTIVITY OF SAMPLES WITH X-RAY WAVEGUIDE LAYERS
}

\author{
J.B. Pelka ${ }^{a}$, S. Lagomarsino ${ }^{b}$, S. Di Fonzo ${ }^{c}$, W. Jark ${ }^{c}$ \\ AND J. Domagaea ${ }^{a}$ \\ ${ }^{a}$ Institute of Physics, Polish Academy of Sciences \\ Al. Lotników 32/46, 02-668 Warszawa, Poland \\ ${ }^{b}$ Istituto di Elettronica dello Stato Solido CNR, V. Cineto Romano 42, Roma, Italy \\ ${ }^{c}$ Sincrotrone Trieste, Padriciano 99, 34012 Trieste, Italy
}

The glancing-angle reflectivity profiles in samples containing an X-ray waveguide layer are studied. Oscillations observed at angles within the region of resonance and above, are interpreted by angle dependent interference of the monochromatic X-ray beam in thin layer. The discussion is extended to the structures composed of more than one layer. Experimental reflectivity spectra recorded with $\mathrm{Cu} K_{\alpha}$ radiation are compared with the theoretical calculations. It leads to the model of oscillations in reflectivity consistent both for the resonant and non-resonant regions, and clarifies interpretation of oscillations in the region above the resonances. A brief discussion of potential applications of the reflectivity spectra to the studies of structure of thin layers is done.

PACS numbers: $68.65 .+\mathrm{g}, 78.20 . \mathrm{Ci}$

\section{Introduction}

The resonance-enhanced propagation of the X-rays in thin films deposited on an X-ray reflecting mirror was anticipated theoretically in 1991 by Wang et al. [1] and also by de Boer [2]. A year later the effect was experimentally confirmed in an organic thin film deposited on gold mirror by Wang et al., who used a synchrotron radiation from hard X-ray wiggler [3]. Recently, the resonant effects have been found also in thin carbon layers using the conventional X-ray tubes as radiation sources $[4,5]$. The effect of resonance observed in these experiments strongly enhances the electric field inside the film, forming the X-ray standing waves of variable period, depending on the angle of incidence of the primary beam. The spectra of secondary radiation, like fluorescence or photoelectrons excited by this enhanced $E$-field were found in the cited papers to be a useful tool for studying various aspects of the layered structures. The layers that obey conditions for the effect of resonance are called the X-ray waveguide layers due to their similarity to the conventional optical layered waveguide. The resonance-enhanced propagation 
of the X-rays influences significantly in a characteristic way the reflectivity profiles of the samples. Main aim of this work is to study the details of oscillation structure in the reflectivity spectra from samples including the waveguide layers. From the experimental point of view it is much easier to measure the reflectivity rather than the secondary spectra from the samples. Therefore, precise understanding of the details observed in the reflectivity spectra can be of primary importance for fast tests of thin films.

\section{Experimental}

For the reflectivity measurements we used two samples (\#2 and \#3) which were composed of $98.5 \mathrm{~nm}$ carbon film on top of $82 \mathrm{~nm}$ Ni layer which was deposited on the glass substrate of $70 \times 21 \times 4 \mathrm{~mm}^{3}$. In the carbon film of each sample there was deposited an ultra-thin layer of Ti. In sample \#2 it was $0.5 \mathrm{~nm}$ thick, and deposited exactly in center of $\mathrm{C}$ film whereas in sample \#3 its thickness was only $0.2 \mathrm{~nm}$, and position $1 / 3$ of $\mathrm{C}$ layer thickness from the top $\mathrm{C} /$ air interface. The samples were produced by sputtering deposition in the multilayer laboratory of the Sincrotrone Trieste. Sputtering was achieved by use of a triode assisted and magnetically confined plasma in a low pressure Ar atmosphere $(p=0.1 \mathrm{~Pa})$ [4]. The roughness of the sample surface and all interfaces was estimated to be about $0.7 \mathrm{~nm}$ root mean square. The reflectivity measurements were recorded at the Institute of Physics, Polish Academy of Sciences, Warsaw with the $\mathrm{Cu} K_{\alpha}$ radiation by high performance MRD Phillips spectrometer equipped with Si (440) Bartels monochromator and $\mathrm{Ge}(1-1)$ analyzer. The results of these measurements are shown in Fig. 2 (sample \#2) and Fig. 1 (Exp) (sample \#3).

\section{Theoretical approach}

For theoretical calculations of glancing-angle reflectivity spectra we used a computational approach based on the Fresnel equations. The reflectivity of $N$-layers system was calculated taking into account all reflection and transmission processes of a monochromatic incident plane wave at the $N+1$ interfaces of the system. Both for the single layer, and for a more complex system composed of several layers the same basic Fresnel equations can be applied recursively starting from the substrate toward the top interface [1]. In the recursion process for each interface the reflection coefficients and the relative phase shifts are obtained allowing the calculation of the $E$-field distribution in the sample. The same algorithm for the calculations of fluorescence radiation yield excited by the $E$-field in waveguide layers was also used in papers $[4,5]$. The results of the theoretical calculations assuming the monochromatic $\mathrm{Cu} K_{\alpha}$ radiation, and all interfacial roughnesses of 0.7 root mean square are collected in Fig. 1 . The left part of this figure shows the reflectivity profiles at angles above the region of resonance, with oscillation structure described first by Kiessig [7], known also as Parratt's oscillations [6].

When the monochromatic $\mathrm{X}$-ray beam impinges on solid surface at an angle lower than a critical one, $\theta_{\mathrm{c} 1}$, which depends on the quantum energy and on the nature of the reflecting surface, it is totally reflected $[1,6,7]$. If the solid is thick enough, the reflectivity of such "semi-infinite" mirror as a function of an angle slowly monotonically decreases up to the critical angle, and at steeper angles it 

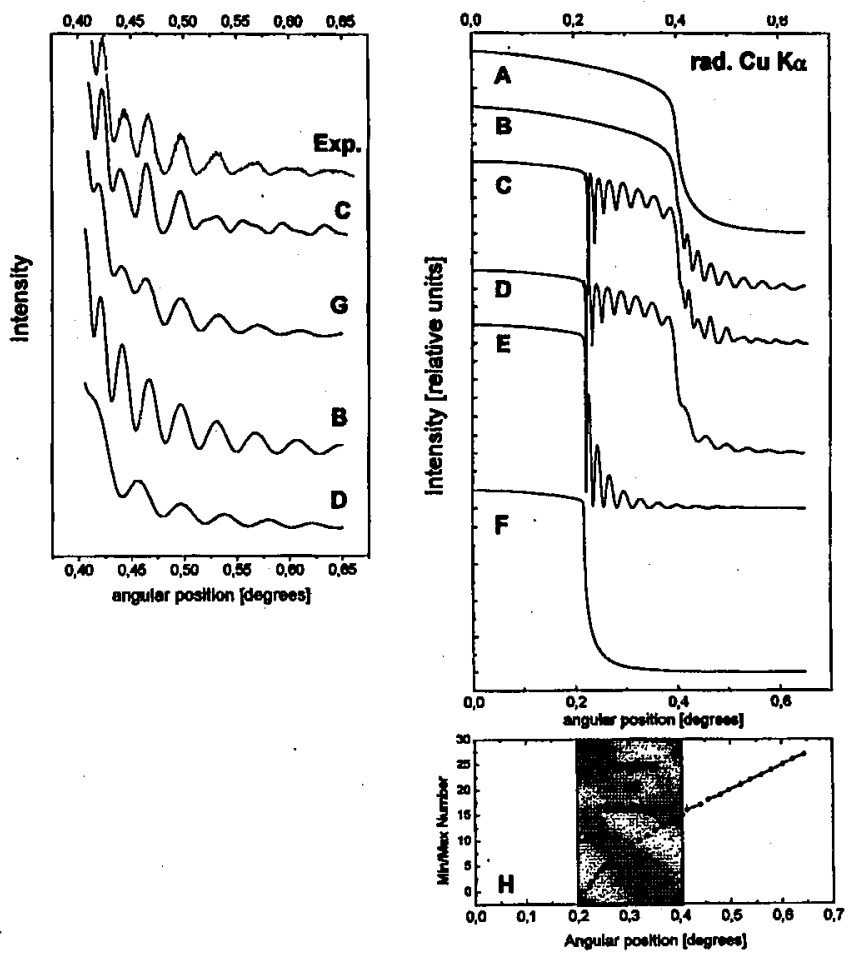

Fig. 1. Theoretical calculations of glancing-angle reflectivity from samples with various layer structure. See text for explanation. Calculated reflectivity: A - Ni bulk mirror, $\mathrm{B}-82 \mathrm{~nm}$ thick $\mathrm{Ni}$ mirror, $\mathrm{C}-98.5 \mathrm{~nm} \mathrm{C}$ on $82 \mathrm{~nm} \mathrm{Ni}$ (sample \#3), D $-98.5 \mathrm{~nm} \mathrm{C}$ layer on top of $\mathrm{Ni}$ bulk, $\mathrm{E}-98.5 \mathrm{~nm} \mathrm{C}$ on top of the surface with $Z=2, \mathrm{~F}-$ carbon bulk mirror, $\mathrm{G}$ - sum of curves $\mathrm{B}$ and $\mathrm{D}, \mathrm{H}$ - positions of the minima and maxima in $98.5 \mathrm{~nm}^{2} \mathrm{C}$ layer on $\mathrm{Ni}$ bulk, Exp. - experimental reflectivity (sample \#3).

reaches fast near-zero values (Fig. 1, A, F). A standing wave field is formed above the reflecting surface with a periodicity $D$ which depends on the incident angle and on the wavelength: $D=\lambda / 2 \sin \theta$. As the $\theta_{c 1}=\sqrt{2 \delta}$, where $\delta$ is the real part of the refractive index for the material, the total external reflection region under this critical angle is for all materials very small. If the mirror is constituted of a thin overlayer film, above the critical angle there are observed oscillations, due to the interference between the beams reflected at the film surface and at the film-substrate interface. The oscillation period provides information on the film thickness. The positions of a set of minima and maxima can be found from equation describing the difference of optical path for the X-ray beams interfering in thin layer

$$
2 d R \sin \theta=2 d(1-\delta) \sqrt{\sin ^{2} \theta_{0}(1+2 \delta)-2 \delta}=n \lambda,
$$

here $R$ is the refraction index, $d-$ layer thickness, $\theta_{0}-$ angle of incidence of the primary beam, and $\theta-$ angle of refraction. Neglecting the $\delta$ values, with $R=1$, 
$\theta=\theta_{0}$ and replacing $\sin \theta$ with the $\theta$ value, we obtain the following formula for the $\theta_{n}$ positions of the interference maxima and minima above the critical angle $\theta_{\mathrm{c}}$ in thin layer

$$
2 d \sqrt{\theta_{n}^{2}-\dot{\theta}_{\mathrm{c}}^{2}}=n \lambda / 2 \text {; with } n \text { odd for minima and } n \text { even for maxima. }
$$

If a thin film is deposited on the reflecting material characterized by $\theta_{\mathrm{c} 2}>\theta_{\mathrm{c} 1}$, the incoming beam will penetrate through this layer, but it will be totally reflected by the underlying mirror. This phenomenon can take place within the angles of incidence between the $\theta_{\mathrm{c} 1}$ and $\theta_{\mathrm{c} 2}$, and it leads to the considerable enhancement of the $E$-field intensity in the 0layer [3,4]. Also here a set of oscillations occurs, which can be, however, much deeper comparing to the previous ones. The positions of "resonant" oscillations, and that of above the region of resonance belong to the same system of oscillations. This is illustrated in Fig. 1, H, where we can see the inverse-parabolic dependence of the maxima and minima positions in the calculated reflectivity from the waveguide layer, according to $\mathrm{Eq}$. (1b).

When the underlying mirror is also constituted of thin film, it can produce another system of oscillations. The both systems of oscillations, from the waveguide film and from the mirror layer contribute to the reflectivity above the $\theta_{\mathrm{c} 2}$. In order to learn how approximation coming from simple superposition of two sets of oscillations explains the real case, the reflectivity model calculated for the sample \#3 (Fig. 1, C) was compared with the curve calculated by adding the oscillation intensities from single carbon layer (Fig. 1, D), and from the single $\mathrm{Ni}$ mirror (Fig. 1, B). The result is shown in Fig. 1, G. Clearly the structure of first four maxima in the region above the resonance, can be related to the calculated "true" structure from Fig. 1, C. The intensity relations of the four peaks are highly similar. However, for the next peaks this simple model does not work well, and traces of oscillations with other period are visible. The comparison of this experimental curve (Fig. 1, Exp) with the presented calculations indicates that the oscillations coming from Ni layer contribute slightly stronger to the reflectivity above the region of resonance than it is predicted by theory. The reason for this is not quite clear; it can follow the fact that the influence of the roughness increases with angle in the glancing-angle reflectivity, which can suppress the oscillations from the carbon layer at steeper angles [6].

\section{Results and discussion}

In Fig. 2 there is shown comparison of the experimental (in the linear and logarithmic scale) and the theoretical reflectivity for the sample \#2. The agreement between the curves is very good concerning the position of the resonances, and satisfactory concerning the general shapes. In the region of resonance seven positions of resonances are identified, indicated by the angular positions of reflectivity minima. At angles steeper than the critical one for the $\mathrm{C} / \mathrm{Ni}$ interface, the reflectivity decreases in intensity. The amplitude of oscillations also decreases slowly with an angle. The depth in the even resonant minima in experimental curve is clearly higher, comparing to that in odd ones. This discrepancy almost vanishes when we assume in the calculations smaller thickness of Ti. The best fit to the experimental data was obtained when assuming the Ti layer thickness 

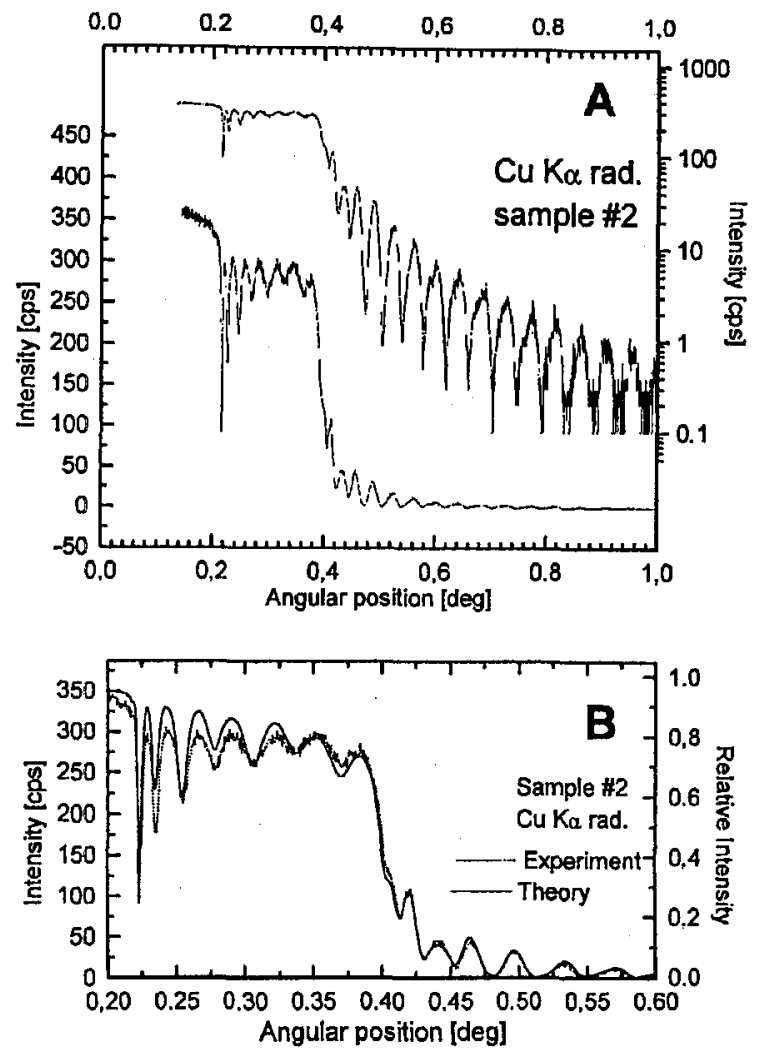

Fig. 2. Experimental reflectivity of the sample \#2 recorded with $\mathrm{Cu} K_{\alpha}$ radiation shown in linear and logarithmic intensity scale (A); comparison of experimental and theoretical intensity (B).

in the range of $0.2-0.3 \mathrm{~nm}$. This also shows how shape of the reflectivity itself, and particularly the intensity ratios among the peaks, can be sensitive to details of sample composition. However, in this particular case the reason of this discrepancy can be connected with inhomogeneity of the Ti layer rather than with lowering in its thickness. An importance of this factor is clear if we compare the roughness of the interfaces $(0.7 \mathrm{~nm})$ and the thickness of the Ti layer $(0.5 \mathrm{~nm})$. The result of theoretical calculation can be an indication that in case of perfect homogeneous Ti layer, the reflectivity from the sample \#2 could be better explained as a system of two equally thick $\mathrm{C}$ layers instead of just one layer. The theoretical reflectivity falls down slightly faster at higher angles within the region of resonance than it is observed in experimental data. It can be an indication that the roughness in the calculations is slightly overestimated.

The accuracy of evaluation of the layer thickness by fitting the theoretical model to the experimental reflectivity was estimated for the cases studied in this paper to be better than $0.3 \%$ for the $\mathrm{C}$ layer, and better than $1 \%$ for the Ni layer. 


\section{Conclusions}

Properties of the X-ray reflectivity of samples with resonance-enhanced propagation of the beam in thin film are very useful for study of structures and various processes in thin layers. It is also possible to extract information about the surface and interface roughness of the sample by comparison of experimental data with the theoretical model. The applicability of the phenomenon exceeds far beyond the solid inorganic films; it can be applied also in studies of structure and processes of organic or liquid films of the thickness of the order of tens-hundreds nanometers. Oscillations observed at angles within the region of resonance and above, can be interpreted by angle dependent interference of the monochromatic X-ray beam in thin layer. It leads to the model of oscillations in reflectivity consistent both for the resonant and non-resonant regions, and clarifies interpretation of oscillations in the region above the resonances. In the structures composed of more than one layer the oscillations coming from each layer contribute to the reflectivity above the region of resonance. An existence of the resonant oscillations in the reflectivity can simplify the analysis of the composed oscillation system, because the resonant oscillations are very well defined in position, and therefore it should be helpful in extraction of this type of oscillations from the oscillation structure above the region of resonance. This simple model does not include the beam interactions coming from the different layers. Therefore some additional contribution to the reflectivity structure can be expected especially at higher angles. Comparison of the theoretical and experimental reflectivity recorded with $\mathrm{Cu} K_{\alpha}$ radiation shows generally very good agreement. However, the inftuence of roughness from particular interfaces on the details of reflectivity spectra needs still some theoretical and experimental effort.

\section{Acknowledgments}

This work was supported in part by the Committee for Scientific Research, grant No. 2P03B 02109 . The Phillips MRD diffractometer used in this work was founded by the Foundation for Polish Science (within the program SEZAM 94).

\section{References}

[1] J. Wang, M.J. Bedzyk, T.L. Penner, M. Caffrey, Nature 354, 377 (1991).

[2] D.K.G. de Boer, Phys. Rev. B 44, 498 (1991).

[3] J. Wang, M.J. Bedzyk, M. Caffrey, Science 258, 775 (1992).

[4] S. Di Fonzo, W. Jark, S. Lagomarsino, A. Cedola, B. Muller, J.B. Pełka, Thin Solid Films, accepted for publication.

[5] J.B. Pełka, S. Lagomarsino, A. Cedola, S. Di Fonzo, W. Jark, B. Muller, J. Domagała, to be published in: Zastosowanie Promieniowania Synchrotronowego (Proc. 3rd. Nat. Seminar. Synchr. Rad. Users, Warsaw, 6-7 June 1995), Ed. E. Sobczak, Wydawnictwo Fundacji im. Wojciecha Świętosławskiego, Warszawa 1995, p. 130.

[6] L.G. Parratt, Phys. Rev. 95, 359 (1954).

[7] H. Kiessig, Ann. Phys. (Germany) 10, 715, 769 (1931). 